\title{
Identification of Dynamical Systems Using GMM with VQ Initialization
}

\author{
Jing Lan, Jose C. Principe and *Mark A. Motter \\ Computational NeuroEngineering Lab., University of Florida, Gainesville, FL 32611 \\ *Dynamics and Control Branch, NASA Langley Research Center, Hampton, VA 23681
}

\begin{abstract}
We are using Gaussian Mixture Models (GMM) as a tool to construct local mappings of nonlinear Multi-Input Multi-Output (MIMO) systems. In this work we combine the advantages of GMM with the Kalman filter. To improve the accuracy of the local linear mappings in a potentially large dimensional state space, we propose to initialize the GMM parameters with Vector Quantization (VQ) or its more parsimonious counterpart Growing Self-Organizing Maps (GSOM). The performance of the proposed modeling algorithms on simulated data obtained from a realistic aircraft model show improvements in both converge speed and accuracy.
\end{abstract}

\section{INTRODUCTION}

Local Linear Modeling (LLM) is a basic dynamic modeling methodology [1]. In most realistic conditions, the dynamic equations describing the nonlinear system are unknown. The designer has only access to samples from the input-output data streams. Thus the task of dynamic modeling is to find a local linear model $\tilde{f}$ as an approximation of the unknown functional model $f$ that preserves the dynamical properties [1]. LLM can be considered as a linearization of a nonlinear system. In fact, a time-varying linear predictive model of a signal can generate arbitrarily complex outputs by convolving input data with time varying coefficients. However, in local linear modeling, we use a finite number of models to approximate the system, that is, we are quantizing and limiting the variability of the coefficients over time.

Gaussian Mixture Models are flexible and powerful modeling tools for multivariate data, which also provide promising alternatives for the processing of nonlinear signals. The usefulness of mixture models in many areas of statistical modeling and system identification is widely acknowledged [4].

In order to implement LLM with GMM, several Gaussian models are directly fit to the system input data (unsupervised learning). Once the training of the GMM is done, the fitting of the nonlinear system output $y(k)$ can be simplified to a set of linear coefficient models, which use a weighted summation (by probability $p_{i}$ ) over a set of prediction results $y_{i}(k)$ from each of the Gaussian models,

$$
y(k)=\sum_{i=1}^{N} p_{i} \cdot y_{i}(k)
$$

In this paper, following the work of [3], a GMM is utilized to cluster the non-linear dynamics in state space using the E-M algorithm, where each multi-dimensional

This work was partially supported by Accurate Automation Corporation under grant \#463, and NASA grant NAG-1-02068.
Gaussian model represents a center of a cluster. The Kalman filters are used to calculate the coefficients of the local linear model of each cluster. To overcome the slow convergence of the standard GMM algorithm, improved methods for initializing the parameters based on VQ and G-SOM are developed, and the respective performance is compared. The proposed system identification scheme was tested using data obtained from LoFLYTE, a realistic aircraft model.

\section{SOFT-COMPETITION DyNAMIC MODELING}

\section{A. Description of the system}

The LoFLYTE ${ }^{\circledR}$ aircraft testbed designed by Accurate Automation Corporation is used to demonstrate the versatility of the proposed algorithm in system identification [1].

The longitudinal motion consists of axial $(x)$, vertical $(z)$ and pitch $(q)$, while the lateral motion consists of roll $(p)$, yaw $(r)$ and lateral $(y)$ movements. The elevator and throttle control the longitudinal motion, while the aileron and rudder primarily affect lateral motion. The resulting linearized equations of motion are sixth-order, representing the perturbations in longitudinal and lateral motions. Here we wish to estimate the aircraft's parameters of longitudinal and lateral motions. In general, the dynamics of the system are described by its state dynamic equations and the output mapping [1]. Define

$\vec{x}(k)=\left[\begin{array}{c}p(k), \mathrm{rad} / \mathrm{sec} \\ r(k), \mathrm{rad} / \mathrm{sec} \\ v(k), \text { feet } / \mathrm{sec} \\ q(k), \mathrm{rad} / \mathrm{sec} \\ u(k), \mathrm{feet} / \mathrm{sec} \\ w(k), \text { feet } / \mathrm{sec}\end{array}\right], \vec{u}(k)=\left[\begin{array}{c}\text { aileron angle in degrees } \\ \text { rudder angle in degrees } \\ \text { elevator angle in degrees } \\ \text { throttle }\end{array}\right]$.

Then,

$$
\begin{aligned}
& \vec{x}(k+l)=f(\vec{x}(k), \vec{u}(k)) \\
& \vec{y}(k)=h(\vec{x}(k), \vec{u}(k))
\end{aligned}
$$

\section{B. Gaussian Mixture Model}

The GMM consists of $N$ expert systems (i.e. clusters) and an integrating network, which is referred to as the gating network [3]. Each of the mixture models is usually used in unsupervised learning problems where different domains of the feature space have different output characteristics. The role of the GMM is to divide the input space into a set of 
regions represented by the weight parameters of each cluster according to the LLM methodology.

We construct a LLM from the system I/O data pairs $(\vec{x}, \vec{u})$. Each processing element (PE) in GMM represents a distribution cluster, and $p(\vec{x}, \vec{u})$ is parameterized as a Gaussian mixture density and expanded in a sum over clusters $c_{i}$.

$$
p(\vec{x}, \vec{u})=\sum_{i=1}^{N} p\left(\vec{x}, \vec{u}, c_{i}\right)
$$

Each cluster has an input distribution, a local model, and output distribution. In this way, we cluster state space through competitive learning such that the trained Gaussian model bears a strong local resemblance to the input space [3]. Therefore the GMM is employed as a modeling infrastructure to construct the local linear model. Because of the high dimension of training data, however, GMM is sensitive to its initial parameters. Moreover it is difficult to choose the initial cluster weights of a GMM in advance without any priori information, and a good final prediction performance could only be obtained after many samples by chance.

To overcome this deficiency, we propose to employ Vector Quantization concepts. The Voronoi regions play the role of GMM's clusters. The growing SOM (G-SOM), because of its flexibility for expansion and accuracy, is used as our vector quantizer. Since G-SOM has a constant adaptation parameter for the winning PE, it can approximate the distribution of state space in a stabler manner. The proposed nonlinear modeling scenario is constructed in four steps:

1)Embedding the time series of system dynamics

2) Using VQ/G-SOM get the initial weights of GMM

3)Training the GMM using E-M algorithms

4)Estimating the Local Linear Model using Kalman filters

\section{Methodology}

\section{A. Embedding the time series}

When working with experimental data, we are restricted to observe the system outputs and infer the dynamics from the observation. According to Taken's Embedding Theorem and its generalization [1], the time delay embedding, which is formed by time-delayed values of the measurements,

$$
\overrightarrow{\mathrm{c}}(k)=[\vec{c}(k), \vec{c}(k-\tau), \cdots, \vec{c}(k-(M-l) \tau)]
$$

provides a one-to-one image of the original set, provided the embedding dimension, $M$, is large enough. In MIMO system identification the choice of the embedding plays an important role in defining the representation space.

\section{B. E-M Algorithm}

The standard method used to fit a GMM to data is the E$\mathrm{M}$ algorithm, which converges to a maximum likelihood (ML) estimate of the Gaussian model parameters. Given the observed data as known states, the E-M assumes the known states and unknown states characterizing the whole system model. The E-M algorithm is a recursive procedure, which maximizes the likelihood of current input given the clusters' distribution.

The E-M algorithm produces a sequence of cluster's estimation by recursively applying two steps:

- E-step: Compute the conditional probability that relates each cluster to each data assuming the current cluster parameters are correct.

$$
p\left(c_{i} \mid y, x\right)=\frac{p\left(y, x \mid c_{i}\right) p\left(c_{i}\right)}{\sum_{l=1}^{N} p\left(y, x \mid c_{l}\right) p\left(c_{l}\right)}
$$

The sum in the denominator causes clusters to interact over input data points and specialize in data they explain best. - M-Step: find the cluster parameters that maximize the likelihood of the data assuming that the current data distribution is correct. The new cluster probability $p\left(c_{i}\right)$ is

$$
\begin{aligned}
p\left(c_{i}\right) & =\int p\left(c_{i} \mid y, x\right) p(y, x) d y d x \\
& \approx \frac{1}{N} \sum_{n=1}^{N} p\left(c_{i} \mid y_{n}, x_{n}\right)
\end{aligned}
$$

Once the E-M is done, the conditional probability $p\left(y, x \mid c_{i}\right)$, which relates each data to each cluster is available by means of Bayesian Equation, and it can be used in the prediction stage to weight the prediction result from each local linear model.

\section{Training of G-SOM}

The initial topology of the G-SOM network is a triangle with 3 PEs. During the self-organizing process, one new PE will be added to the map at the end of each training iteration. Every modification of the network is performed such that the updated network consists solely of a 2-D topology and triangular neighborhoods [1]. The adaptation in our model can be formulated as:

1)Locate the winning PE, $s=\min [\operatorname{dist}(\overrightarrow{\mathrm{c}}-\mathrm{w})]$

2)Increase matching for $s$ and its direct neighbors $N_{s}$ $\Delta w_{S}=\varepsilon_{b}\left(\overrightarrow{\mathrm{c}}-w_{S}\right), \Delta w_{c}=\varepsilon_{n}\left(\overrightarrow{\mathrm{c}}-w_{c}\right)$ (for all $c \in N_{s}$ )

3)Increase the winning frequency counter $\tau_{s}$ of $s$ with $\Delta \tau_{s}=1$

4)Decrease all signals' $\tau_{s}$ counters by a small value so that the mean of winning frequency doesn't change too much;

5)After feeding in all data, one new $P E$ is added between the PE with the highest $\tau_{s}$ and its farthest neighbor.

Our objective is a structure with the weights $w_{c}$ distributed according to the distribution of data. When we insert a new PE $r$, it is connected to the others in such a way that we once again preserve the previous structure. The insertion of $r$ leads to a new Voronoi region $F_{r}$ in the state 

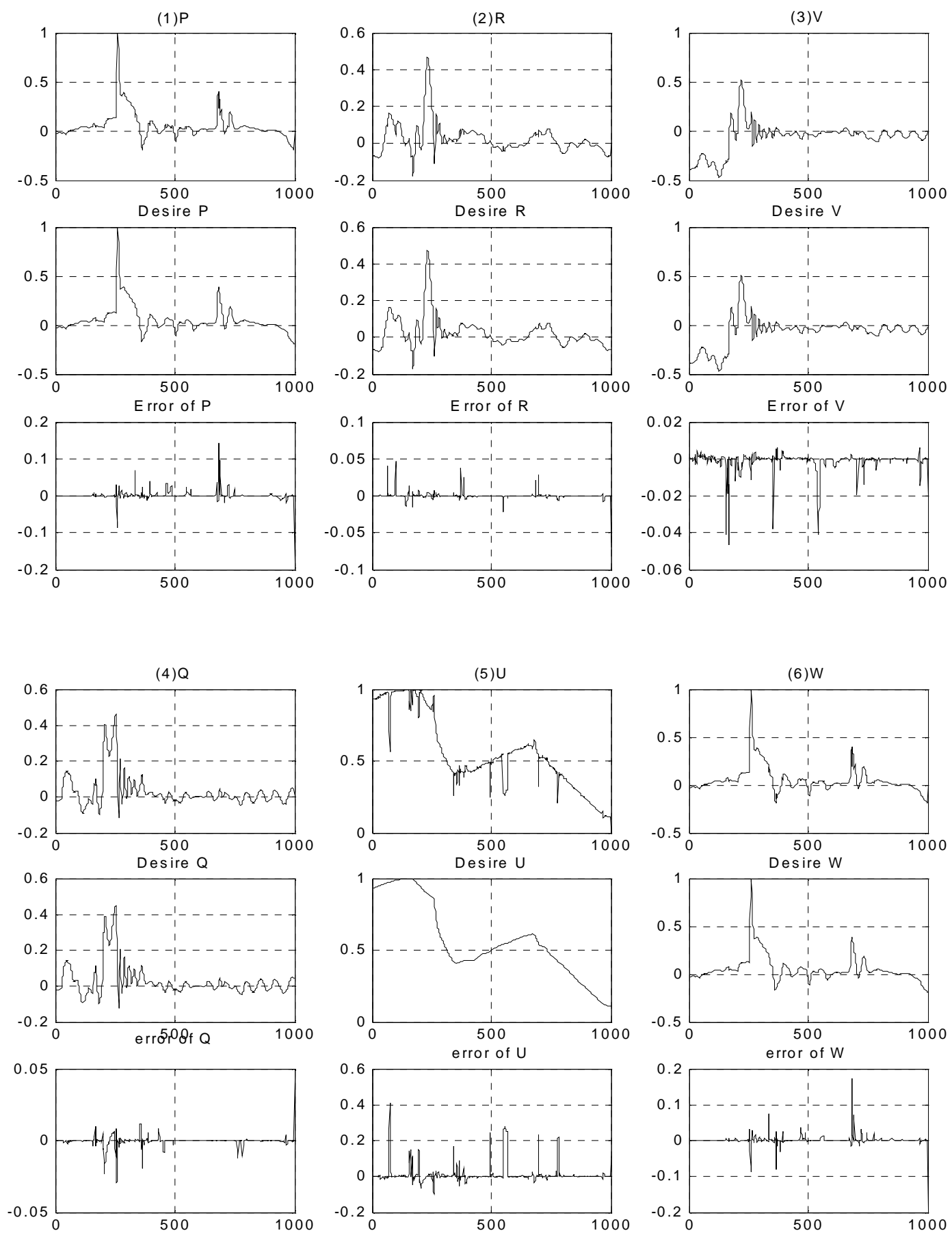

Figure 1. Parameter estimations and error levels.

space. At the same time the Voronoi regions of the topological neighbors of $r$ are diminished [1].

D. Estimating the LLM using Kalman Filter
To estimate the output coefficients $\vec{a}_{i}$, the state space could be represented as

$$
\begin{aligned}
& \vec{a}_{i}[N+1]=E_{i} \cdot \vec{a}_{i}[N]+F_{i} \cdot v_{i}[N] \\
& y_{i}[N]=\vec{H} \cdot \vec{a}_{i}[N]+w[N]
\end{aligned}
$$


TABLE 1. SER PERFORMANCE

where $\quad E_{i}=F_{i}=I ; \vec{H}_{i}=\left[\begin{array}{ll}1 & x^{T}\end{array}\right]$.

Here, $x$ is the input vector as opposed to the state vector of the filter. [3] $v_{i}$ and $w$ are white Gaussian noise. Both noise terms relate to the update of the error covariance matrices associated with a cluster. The assumption of Gaussian distribution is not necessary but a reasonable working hypothesis. The system's state $a_{i}$ is updated to minimize the error covariance of the prediction $y$, taking into account the posterior probability of the new data owned by cluster $c_{i}$. The solution to the problem has the form

$$
\begin{aligned}
\vec{a}_{i}[N \mid N] & =\vec{a}_{i}[N \mid N-1]+G_{i}[N] \\
& \cdot\left(y[N]-H_{i}[N] \vec{a}[N \mid N-1]\right)
\end{aligned}
$$

where $G_{i}[N]$ is the Kalman gain matrix.

The filter updates could be summarized as:

1)Initialize the estimators $\vec{a}[0 \mid-1]=0 ; \Lambda_{e}[0 \mid-1]=\Lambda$ where $\Lambda_{e}$ is the error coefficient covariance.

2)Update the cluster parameters using E-M;

3)Compute the Kalman gain matrices $G_{i}[N]$, weighted by the posterior probability of cluster $c_{i}$ given data $N$, then update $\vec{a}_{i}$

$$
\begin{aligned}
& G_{i}[N]=\Lambda_{e, i}[N \mid N-1] H^{T}[N] \\
& \quad \cdot\left(H[N] \Lambda_{e, i}[N \mid N-1] H^{T}[N]+\Lambda_{w, i}[N]\right)^{-1} \\
& \quad \cdot p^{(N)}\left(c_{k} \mid x_{N}, y_{N}\right) \\
& \vec{a}_{i}[N \mid N]=\vec{a}_{i}[N \mid N-1] \\
& \quad+G_{i}[N] \cdot\left(y[N]-H[N] \cdot \vec{a}_{i}[N \mid N-1]\right) \\
& \Lambda_{e, i}[N \mid N]=\Lambda_{e, i}[N \mid N-1] \\
& \left.\quad-G_{i}[N] \cdot H[N] \cdot \Lambda_{e, i}[N \mid N-1]\right)
\end{aligned}
$$

4)Predict new vector of coefficients $\vec{a}_{i}$

$$
\vec{a}_{i}[N+1 \mid N]=\vec{a}_{i}[N]
$$

5)Increase $N$, go to step 2 [4].

\section{SimUlation RESUlts}

The simulation results were obtained from the application of the proposed algorithm to the identification of a LoFLYTE aircraft system.

After experimentation, a total of 15 clusters were found adequate to represent the dynamics of the flight envelope for the LoFLYTE. We selected an embedding dimension of $N=3$ for the 6-D states and $N=1$ for the 4-D control inputs. Thus the total input dimension is 32 . The G-SOM is trained with 3000 samples for 15 epochs.

To explore the usefulness of a VQ front-end to initialize the GMM clusters, the experiment is carried out using a 15PE standard K-means clustering, growing a 15 PE G-SOM and GMM without any preprocessor. All other training conditions are kept the same.

The trained GMM are tested on new 800 samples for all outputs. We present in figure 1 the actual and identified

\begin{tabular}{|c|c|c|c|}
\hline \multicolumn{5}{|c|}{20 dB SNR } \\
\hline & GMM & $\begin{array}{c}\text { GMM with K- } \\
\text { mean }\end{array}$ & $\begin{array}{c}\text { GMM with } \\
\text { G-SOM }\end{array}$ \\
\hline P & 15.116 & 16.161 & 15.991 \\
\hline Q & 14.584 & 14.711 & 15.484 \\
\hline R & 14.978 & 15.704 & 15.232 \\
\hline U & 11.924 & 15.057 & 14.888 \\
\hline V & 14.921 & 16.538 & 16.597 \\
\hline W & 15.011 & 14.391 & 14.732 \\
\hline Average & $\mathbf{1 4 . 4 2 2}$ & $\mathbf{1 5 . 4 2 7}$ & $\mathbf{1 5 . 4 8 7}$ \\
\hline \multicolumn{5}{|c|}{40 dB SNR } \\
\hline P & 23.637 & 23.391 & 23.405 \\
\hline Q & 26.012 & 24.941 & 24.396 \\
\hline R & 24.663 & 23.534 & 22.553 \\
\hline U & 21.016 & 25.387 & 26.352 \\
\hline V & 22.682 & 28.585 & 28.544 \\
\hline W & 22.829 & 21.932 & 25.668 \\
\hline Average & $\mathbf{2 3 . 4 7 3}$ & $\mathbf{2 4 . 6 2 8}$ & $\mathbf{2 5 . 1 5 3}$ \\
\hline \multicolumn{5}{|c|}{0 Noise } \\
\hline P & 25.514 & 23.390 & $\mathbf{2 4 . 7 2 1}$ \\
\hline Q & 27.828 & 24.545 & $\mathbf{2 5 . 8 3 8}$ \\
\hline R & 22.053 & 24.316 & $\mathbf{2 5 . 6 9 6}$ \\
\hline U & 19.065 & 25.744 & $\mathbf{2 7 . 1 2 3}$ \\
\hline V & 26.530 & 28.700 & $\mathbf{2 9 . 4 4 4}$ \\
\hline W & 23.315 & 22.920 & $\mathbf{2 5 . 8 5 3}$ \\
\hline Average & $\mathbf{2 4 . 0 5 1}$ & $\mathbf{2 4 . 9 3 6}$ & $\mathbf{2 6 . 4 4 6}$ \\
\hline
\end{tabular}

trajectory of the system and the corresponding errors using GMM combining G-SOM. In figure 1, the first and forth rows plot predicted results from testing data. In the second and fifth rows, true values are shown. And in the third and the sixth rows, prediction errors are provided for the same data. The figures show that the system has large dynamic range and the prediction is generally close to the actual output with small error. The error dynamic range is most often $25 \%$ of the signal (except for ' $U$ '), and it is limited to the fast changes in dynamics.

We tabulate in table 1 the improvement in terms of signal to error ratio (SER) in $\mathrm{dB}$. It is clear that the results from GMM with the K-mean and G-SOM have higher accuracy than GMM alone especially on parameters ' $U$ ' and ' $V$ '. The identified output equals the actual output except at those points, which are not described well by training data. For some parameters, take ' $U$ ' for example, its dynamics doesn't change as quickly as others do. In a 32-dimension embedding space, some of the testing data cannot take any of the GMM clusters as estimation winner with large probability, in that case the prediction from the GMM will have larger error rate than other methods.

Training clusters with G-SOM first, however, can make use of the advantage of G-SOM that the distribution of clusters' weights is determined automatically by the input data. In that way, the GMM's clusters' initial weights will 
cover the whole distribution envelope described by training data set. If there is some testing data that has larger distance to the cluster center, all clusters have a large distance to the data, which will lead to small probability of that data belonging to any of the clusters. Small probability leads to occasional large errors in the prediction results shown in Figure 1. As the number of large error grows, new G-SOM $\mathrm{PE}$ will be added in and that will in turn lead to more GMM clusters.

Theoretically, G-SOM uses the same mechanism as standard K-means vector quantization algorithm. Using standard K-means needs to pre-determine the number of clusters. Using G-SOM, however, the number of clusters could be determined by other criterion, which flexibility would be useful when the training data cannot cover the whole state space and the recursive training is necessary.

\section{CONCLUSIONS}

This paper has made the following contributions:

- First we have combined the superior estimating capability of the GMM with stable initialization capability of G-SOM to generate optimized results for identification.

- Second, we have made use of the simplicity of LLM and Soft-Competition modeling for a real-world flight control project.

GMMs have been widely studied on multiple models for engineering applications. The proposed architecture involves a G-SOM that determines the distribution of the state dynamics of the observed system and selects an appropriate local linear model, which can improve the prediction performance further.

Future work should focus on determining methodologies for the optimal selection of embedding length and delay from data. In addition, the Gaussian mixture model could be relaxed to a generalized Gaussian mixture model for better generalization properties.

\section{REFERENCES}

[1] J.Cho, J. Lan, G. K. Thampi, J. C. Principe and M.A. Motter, "Identification of Aircraft Dynamics Using a SOM and Local Linear Models," Proceedings of MWSCAC, Tulsa, Oklahoma, 2002. http://www.cnel.ufl.edu/files/1032800090.pdf

[2] B. Fritzke, "Growing Cell Structures - A Self-Organizing Network for Unsupervised and Supervised Learning," IEEE Trans. Neural Networks, vol. 7, no. 9, pp. 1441-1460, 1994.

[3] B. Schoner, Probabilistic Characterization and Synthesis of Complex Driven System, Ph.D Dissertation, MIT, Cambridge, MA, 1996.

[4] S. Haykin, Adaptive Filter Theory, $3^{\text {rd }}$ ed., Prentice Hall, Englewood Cliffs, NJ, 1996.

[5] J.C. Principe, L. Wang, and M.A. Motter, "Local Dynamic Modeling with Self-Organizing Maps and Application to Nonlinear System Identification and Control," Proc. of IEEE, vol. 86, No. 11, pp. 2240-2258, 1998.

[6] M. Casdagli, "Nonlinear prediction of chaotic time series," Phys. D, vol. 35, pp.27, 1991.

[7] T. Kohonen, Self-Organizing Maps, $2^{\text {nd }}$ ed., Springer, Berlin, 1997.

[8] T. Martinetz, H.Ritter, and K. Schulten, "Neural-Gas Network for Vector Quantization and its application to Time-Series Prediction," IEEE Trans. Neural Networks, vol. 4, no. 4, pp. 558-568, 1993.

[9] K. Narendra, K. Parthasarathy, "Identification and Control of Dynamical Systems using Neural Networks," IEEE Trans. Neural Networks, vol. 1, no. 1, pp. 4-17, 1997.

[10] J. Walter, H. Ritter, and K. Schulten, "Non-linear Prediction with Self-Organizing Maps," Proceedings of IEEE IJCNN-90, S. Diego, CA, vol. 3, pp. 589-594, 1990.

[11] J. J. K. O Ruanaidh and W. J. Fitzgerald, Numerical Bayesian Methods Applied to Signal Processing, Springer-Verlag, New York, 1995.

[12] B.S. Kim, Anthony J. Calise, "Nonlinear Flight Control Using Neural Networks," Journal of Guidance, Control and Dynamics, vol. 20, no. 1, pp. 26-33 January-February 1997.

[13] S. Akaho, "The EM algorithm for multiple object recognition," Proceedings of IEEE Neural Networks, vol. 5, pp. 2426-2431 November-December 1995. 